\title{
Exceptional response to cetuximab monotherapy in a patient with metastatic oropharyngeal squamous cell carcinoma: a molecular insight
}

This article was published in the following Dove Press journal:

OncoTargets and Therapy

9 February 2016

Number of times this article has been viewed

Prakash Peddi'

Bhavna Paryani

Amol Takalkar ${ }^{2}$

Paige Bundrick ${ }^{3}$

John Ponugupati ${ }^{4}$

Binu Nair ${ }^{5}$

Hazem El-Osta'

'Department of Medicine, Division of Hematology-Oncology, ${ }^{2}$ Department of Radiology, ${ }^{3}$ Department of Head and Neck Surgery, Louisiana State University Health Sciences Center, Shreveport, LA, ${ }^{4}$ Oncology Department, Herbert J Thomas Memorial Hospital, South Charleston, WV, ${ }^{5}$ Baylor Scott \& White Medical Center - Waxahachie, Waxahachie, TX, USA
Correspondence: Hazem El-Osta Department of Medicine, Division of Hematology-Oncology, Louisiana State University Health Sciences Center, I50I Kings Highway, Shreveport, LA 71130 , USA

Tel +I 3186754943

Fax + I 318 813 1007

Email helost@lsuhsc.edu
Background: Metastatic head and neck squamous cell carcinoma (HNSCC) carries a very poor prognosis. A better understanding of the molecular driver of the disease and the identification of biomarkers of response remain paramount for an effective personalized therapy.

Case report: We report an original case of a 56-year-old patient diagnosed with metastatic HNSCC to both kidneys, who experienced a long-lasting complete response to a single-agent cetuximab, a monoclonal antibody-targeting EGFR. Comprehensive multiplatform biomarker analysis of the tumor revealed the presence of phosphatidyl-inositol 3 kinase mutation, EGFR overexpression, and the absence of PD-1/PD-L1 expression. Since PI3K, a downstream effector of EGFR, is activated, the tumor regression may have occurred mainly through a cetuximabinduced immune-mediated response, rather than EGFR signal blockade. It is plausible that this effect was enhanced by the lack of PD-1 and PD-L1 expression.

Conclusion: Our case proposes that the absence of PD-1 and PD-L1 expression in conjunction with EGFR overexpression may correlate with better response to cetuximab in HNSCC. This hypothesis needs to be examined through a large clinical trial.

Keywords: biomarker, cetuximab, EGFR blockade, exceptional response, head and neck squamous cell carcinoma

\section{Introduction}

Head and neck squamous cell carcinomas (HNSCCs) are a heterogeneous group of diseases with a biologically aggressive nature. Palliative chemotherapy remains the standard of care treatment in the metastatic setting, and it is associated with modest response and, in general, poor prognosis. The current molecular classification discerns only the human papillomavirus (HPV)-positive and HPV-negative HNSCCs. Beyond HPV status, the lack of predictive biomarkers remains a major obstacle in the treatment of the disease. ${ }^{1}$

Recently, extensive efforts have been made to incorporate molecular information in order to underpin the molecular driver of the disease, guide treatment decision, and predict outcome. By deciphering the mechanisms of response to targeted therapy, comprehensive genomic profiling of exceptional responders serves as a promising approach that may aid in identifying validated molecular phenotypes of sensitivity to targeted agents that would eventually lead to more effective personalized therapy. ${ }^{2}$

We describe here the clinical course and genomically characterize the tumor profile of a patient with metastatic HNSCC who achieved a unique and durable response to epidermal growth factor receptor (EGFR) monoclonal antibody. Our report illustrates 
a candidate biomarker that may be associated with improved response to cetuximab monotherapy.

\section{Case report}

A 56-year-old woman with a 25-pack-year smoking history and alcohol abuse initially presented with a 12-month history of worsening dysphagia, odynophagia, and unintentional weight loss. Physical examination revealed a hard and fixed right-sided neck mass. Flexible laryngoscopy revealed an asymmetric mass at the base of tongue of which the biopsy was consistent with HPV-negative, moderately differentiated squamous cell carcinoma. Initial 18-fluorodeoxyglucose (FDG) positron emission tomography and computerized tomography (PET/CT) scan revealed an extensive, intensely FDG-avid, right base of tongue lesion and metastatic right upper neck lymphadenopathy with no distant metastasis (Figure 1A). Definitive concurrent chemoradiation was initiated using high-dose cisplatin. The first chemotherapy cycle was complicated by acute renal injury. Therefore, she was switched to cetuximab and received a loading dose of $400 \mathrm{mg} / \mathrm{m}^{2}$ followed by $250 \mathrm{mg} / \mathrm{m}^{2}$ weekly concurrently along with radiation treatment (total dose of $70 \mathrm{~Gy}$; $200 \mathrm{cGy} /$ fraction). She experienced a complete metabolic response on restaging PET/CT at 6 months (Figure 1B). A follow-up PET/CT obtained 19 months from the initial
PET/CT was consistent with the persistent response in the head and neck region but showed an intense right renal lesion and another less intense lesion on the left kidney (Figure 1C). Subsequently, she underwent right partial nephrectomy. Surgical pathology confirmed a moderately differentiated metastatic squamous cell carcinoma with morphology very similar to the previously biopsied tongue lesion indicating the metastasis of head and neck origin. Repeat PET/CT scan performed at 22 months revealed a slight progression of the left renal lesion without any evidence of FDG-avid metastatic disease elsewhere (Figure 1D). She was therefore initiated on palliative cetuximab and achieved complete remission as evidenced by restaging PET/CT 2 months later (Figure 1E). The patient continued therapy for 4 additional months. Cetuximab was subsequently stopped for 6 months as per patient's preference. Interval PET/CT performed at 36 months showed recurrence in the left kidney (Figure 1F). Cetuximab was resumed again and she had the resolution of the prior noted left kidney lesion within 3 months after the resumption of the therapy (Figure 1G). The patient was on intermittent cetuximab therapy with recurrence during break and response during treatment. She eventually underwent stereotactic body radiation therapy to the left kidney lesion 4 years later due to local progression. Maintenance cetuximab was resumed subsequently, and the patient remained on it for 12 additional

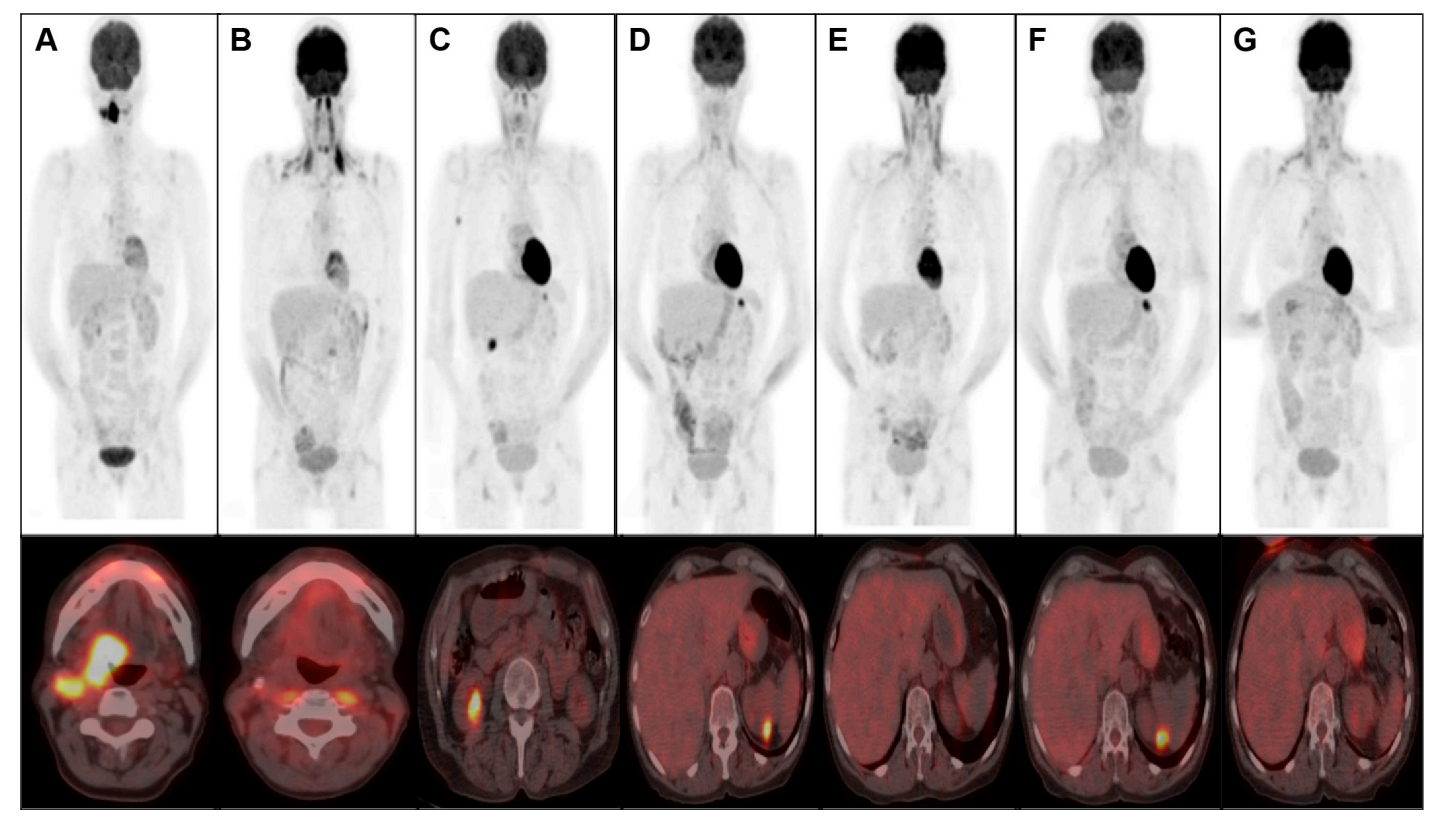

Figure I FDG PET/CT images with upper row showing PET scan sequences and lower row showing respective CT cross-sections.

Notes: (A) Initial PET/CT depicting a locally advanced disease upon diagnosis. (B) Complete remission following definitive concurrent chemoradiation. (C) Appearance of right kidney lesion that turned out to be metastatic squamous cell carcinoma on nephrectomy. (D) Appearance of FDG-avid left renal lesion. (E) Complete metabolic response in the left kidney after the initiation of cetuximab. (F) Relapse in the left kidney after treatment holiday. (G) Complete remission again observed after the reinitiation of cetuximab therapy.

Abbreviations: FDG, fluorodeoxyglucose; PET/CT, positron emission tomography and computerized tomography. 
Table I Summary of molecular profiling of the recurrent tumor performed by CARIS test

\begin{tabular}{|c|c|c|c|}
\hline Involved signaling pathway & Test & Method & Result \\
\hline Tyrosine kinase surface & EGFR expression & $\mathrm{IHC}$ & Positive \\
\hline \multirow[t]{6}{*}{ receptors } & EGFR mutation & NGS & Negative \\
\hline & c-met expression & $\mathrm{IHC}$ & Negative \\
\hline & c-met mutation & NGS & Negative \\
\hline & Her2neu expression & $\mathrm{IHC}$ & Negative \\
\hline & Her2neu amplification & $\mathrm{CISH}$ & Negative \\
\hline & Her2neu mutation & NGS & Negative \\
\hline \multirow[t]{2}{*}{ RAS/RAF/MEK } & HRAS, KRAS, NRAS mutation & NGS & Negative \\
\hline & BRAF mutational analysis & NGS & Negative \\
\hline \multirow[t]{5}{*}{$\mathrm{PI} 3 \mathrm{~K} / \mathrm{AKT} / \mathrm{mTOR}$} & PIK3CA mutation & NGS & Exon 9/E542K Mut \\
\hline & AKTI mutation & NGS & Negative \\
\hline & AKTI expression & $\mathrm{IHC}$ & Negative \\
\hline & PTEN expression & $\mathrm{IHC}$ & Positive \\
\hline & PTEN mutation & NGS & Negative \\
\hline \multirow[t]{2}{*}{ Immune checkpoint } & PD-I expression & $\mathrm{IHC}$ & Negative \\
\hline & PD-LI expression & $\mathrm{IHC}$ & Negative \\
\hline Apoptosis & P53 & NGS & Exon $5 / C I 765$ Mut \\
\hline
\end{tabular}

Abbreviations: $\mathrm{CISH}$, chromogenic in situ hybridization; IHC, immunohistochemistry; NGS, next-generation sequencing; Mut, mutant.

months, experiencing good local control and good tolerance except for grade 1 mucocutaneous toxicity. Comprehensive multiplatform biomarker analysis performed on the left kidney metastasis discovered EGFR overexpression by immunohistochemistry, PI3K E542K mutation at exon 9, and TP53 C176S mutation at exon 5. Neither PD-1/PD-L1 expression nor RAS and BRAF mutations were detected (Table 1). The patient provided a written informed consent for the treatment. Institutional Review Board approval was not sought as the treatment offered is standard of care and not part of a clinical trial therefore considered exempt from approval by the Institutional Review Board of Louisiana State University Health Sciences Centre in Shreveport.

\section{Discussion}

HNSCC is the sixth leading cause of cancer worldwide. ${ }^{1}$ Most patients present with advanced disease at the time of diagnosis, with regional nodal involvement in $43 \%$ and distant metastasis in $10 \%$ of the cases. The most common distant metastatic sites are lung $(80 \%)$, mediastinal lymph nodes $(34 \%)$, bone $(31 \%)$, and liver $(31 \%) .{ }^{3}$ Isolated renal metastasis is very rare and only a limited number of cases have been published in the literature..$^{4-7}$ Amid a complex genetic landscape, metastatic HNSCC carries a very poor prognosis. ${ }^{8}$ A better understanding of the molecular driver of the disease is fundamental for an efficacious personalized treatment. Currently, EGFR is recognized as a critical target in the treatment of HNSCC. It is overexpressed in $>90 \%$ of HNSCC, and it is associated with a poor prognosis. ${ }^{8}$ Cetuximab, a human-murine chimeric immunoglobulin
G1 monoclonal antibody, blocks the EGFR after binding to its extracellular domain. The antitumoral activity of cetuximab occurs via various mechanisms: 1) preventing the ligand transforming growth factor alpha and epidermal growth factor from binding to their receptor (EGFR), thus decreasing the receptor activity and subsequently tumor growth; ${ }^{9}$ 2) downregulating the number of surface EGFR via their internalization and endosomal degradation; ${ }^{9}$ or 3) inducing an immune-mediated response that leads to cancer cell apoptosis. The latter can be the result of direct killing via antibody-dependent cellular cytotoxicity (ADCC) and complement-mediated cytotoxicity, or opsonization of tumor for phagocytosis and subsequent antigen processing by antigen-presenting cells. ${ }^{10}$ Of note, ADCC results from the interaction of the Fc portion of the monoclonal antibody (such as cetuximab) with the Fc receptor on the immune cells, primarily natural killer cells and monocytes.

As demonstrated in the EXTREME trial, the addition of cetuximab to platinum-5-fluorouracil doublet significantly improves the response rate and progression-free and overall survival in metastatic HNSCC. ${ }^{11}$ Cetuximab when used as a single agent exhibits a cytostatic effect rather than cytotoxic effect, with an objective response rate of only $13 \%{ }^{12,13}$ As per our knowledge, only one previous case described a complete and prolonged response to a single-agent cetuximab in metastatic HNSCC. The tumor was positive for HPV and negative for EGFR expression. ${ }^{14}$

Mechanism-based predictors of response to cetuximab in HNSCC remain incompletely characterized, and the discovery of reliable predictive biomarkers employing genomic and 
proteomic analysis is paramount. In metastatic non-small cell lung cancer (NSCLC), FLEX trial has shown that the addition of cetuximab to cisplatin and vinorelbine improves the overall survival in the subgroup of patients overexpressing EGFR. ${ }^{15}$ On the contrary, the value of EGFR overexpression as biomarker of response to EGFR monoclonal antibody in HNSCC has not been established, ${ }^{8}$ In fact, the benefit of adding cetuximab to chemotherapy was observed independently of EGFR expression level. Unlike what is observed in NSCLC, EGFR tyrosine kinase inhibitors have modest clinical efficacy in HNSCC. The discrepancy in the predictive value of EGFR expression along with the differential therapeutic response to a distinct class of EGFR inhibitors may reflect a difference in the dependency to EGFR pathway between HNSCC and NSCLC. Indeed, cetuximab-mediated ADCC may account for the difference in therapeutic activity between tyrosine kinase inhibitor and EGFR monoclonal antibodies in HNSCC and partially explains why biomarkers focused on EGFR expression are not reliable in HNSCC. ${ }^{16}$

Our case supports a cetuximab-dependent immunologic response against the tumor. In our patient, molecular profiling revealed EGFR overexpression. Interestingly, the patient also carries PI3K mutation, known as a mechanism of resistance to EGFR inhibitor through constitutive activation of the downstream EGFR-mediated signaling pathway, AKT/PI3K/mechanistic target of rapamycin, despite the blockage of EGFR. ${ }^{12,17}$ In addition, p53 mutation, a process that renders cancer cells refractory to apoptosis, was found in the tumor, a common finding in HPV-negative HNSCC. The excellent response to cetuximab monotherapy, despite the activation of compensatory escape pathways, can be explained by cetuximab-mediated ADCC rather than EGFR signaling blockage per se. ${ }^{16}$ It is very possible that the absence of PD-1 and PD-L1 expression known as negative regulators of the antitumoral immune response has further potentiated the cetuximab-mediated immune response. ${ }^{8}$ Therefore, this observation goes along with the concept of synergism between tumor-targeting monoclonal antibody and checkpoint inhibitors, such as PD-1/PD-L1 axis blockers. Building on these observations, this case provides some insight about urgently needed biomarkers that predict response to targeted therapy. Since the majority of patients with recurrent metastatic HNSCC express PD-1 and/or PD-L1, ${ }^{18}$ the lack of their expression in the context of EGFR overexpression may define a small subset of patients who can derive clinical benefit from a single-agent cetuximab. Adequate evidence from large genomics databases are required to examine this hypothesis.

\section{Disclosure}

The authors report no conflicts of interest in this work.

\section{References}

1. Suh Y, Amelio I, Urbano G, et al. Clinical update on cancer: molecular oncology of head and neck cancer. Cell Death Dis. 2014;5:e1018.

2. Chen HX, Abrams JS. Genomic profiling: building a continuum from knowledge to care. JAMA Oncol. 2015;1(4):474-475.

3. Kuperman DI, Auethavekiat V, Adkins DR, et al. Squamous cell cancer of the head and neck with distant metastasis at presentation. Head Neck. 2011;33(5):714-718.

4. Wada T, Okuzono T, Okuzono Y, et al. Renal metastasis from squamous cell carcinoma of the hypopharynx. Urol Int. 2002;68(2): $132-134$.

5. Lecoeuvre V, Degardin M, Penel N, et al. Renal colic due to kidney metastase from larynx cancer. Rev Med Interne. 2003;24(1):67-69.

6. Paul NS, Harden P, Fowler R, et al. Renal metastases from a squamous cell carcinoma of the larynx. Br J Radiol. 1999;72:1117-1119.

7. Unni R, Wahle GR, Scott JW. Renal metastasis of head and neck squamous cell carcinoma with inferior vena caval thrombus. J Urol. 2004;171(3): 1236.

8. Sacco A, Cohen E. Current treatment options for recurrent or metastatic head and neck squamous cell carcinoma. J Clin Oncol. 2015;33(29): 33013-33015.

9. Huang SM, Li J, Harari PM, et al. Molecular inhibition of angiogenesis and metastatic potential in human squamous cell carcinoma after epidermal growth factor receptor blockade. Mol Cancer Ther. 2002;1: 507-514.

10. Kimura H, Sakai K, Arao T, Shimoyama T, Tamura T, Nishio K. Antibodydependent cellular cytotoxicity of cetuximab against tumor cells with wild-type or mutant epidermal growth factor receptor. Cancer Sci. 2007;98:1275-1280.

11. Vermorken JB, Mesia R, Rivera F, et al. Platinum-based chemotherapy plus cetuximab in head and neck cancer. N Engl J Med. 2008; 359:1116-1127.

12. Wang Z, Martin D, Molinolo A, et al. mTOR co-targeting in cetuximab resistance in head and neck cancers harboring PIK3CA and $R A S$ mutations. J Natl Cancer Inst. 2014;106(9):1-11.

13. Vermorken JB, Trigo J, Hitt R, et al. Open-label, uncontrolled, multicenter phase II study to evaluate the efficacy and toxicity of cetuximab as a single agent in patients with recurrent and/or metastatic squamous cell carcinoma of the head and neck who failed to respond to platinumbased therapy. J Clin Oncol. 2007;25:2171-2177.

14. Rubinstein PG, Setty S, Lindgren V, et al. Durable complete remission induced by cetuximab monotherapy in a patient infected with HIV and diagnosed with recurrent squamous cell carcinoma of the head and neck. J Clin Oncol. 2011;29(9):e222-e225.

15. Pirker R, Pereira J, Szczesna A, et al. Cetuximab plus chemotherapy in patients with advanced non-small-cell lung cancer (FLEX): an open-label randomized phase III trial. Lancet. 2009;373(9674): $1525-1531$.

16. Hansen A, Siu L. Epidermal growth factor receptor targeting in head and neck cancer: have we been just skimming the surface? J Clin Oncol. 2013;31(11):1381-1383.

17. Young NR, Liu J, Pierce C, et al. Molecular phenotype predicts sensitivity of squamous cell carcinoma of the head and neck epidermal growth factor receptor inhibition. Mol Oncol. 2013;7(3): 359-368.

18. Seiwert TY, Burtness B, Weiss J, et al. A phase Ib study of MK-3475 in patients with human papillomavirus (HPV)-associated and non-HPV associated head and neck (H/N) cancer. J Clin Oncol. 2014;32:5s. 
OncoTargets and Therapy

\section{Publish your work in this journal}

OncoTargets and Therapy is an international, peer-reviewed, open access journal focusing on the pathological basis of all cancers, potential targets for therapy and treatment protocols employed to improve the management of cancer patients. The journal also focuses on the impact of management programs and new therapeutic agents and protocols on

patient perspectives such as quality of life, adherence and satisfaction. The manuscript management system is completely online and includes a very quick and fair peer-review system, which is all easy to use. Visit http://www.dovepress.com/testimonials.php to read real quotes from published authors.

Submit your manuscript here: http://www.dovepress.com/oncotargets-and-therapy-journal 